\title{
THE
}

UNIVERSITY

University of Rhode Island

OF RHODE ISLAND

DigitalCommons@URI

1989

\section{The Quantum Mechanics of Cluster Melting}

\author{
Thomas L. Beck \\ Jimmie D. Doll \\ David L. Freeman \\ University of Rhode Island, dfreeman@uri.edu
}

Follow this and additional works at: https://digitalcommons.uri.edu/chm_facpubs

Terms of Use

All rights reserved under copyright.

\section{Citation/Publisher Attribution}

Beck, T. L., Doll, J. D., \& Freeman, D. L. (1989). The Quantum Mechanics of Cluster Melting. Journal of Chemical Physics, 90(10), 5651-5656. doi: 10.1063/1.456687

Available at: http://dx.doi.org/10.1063/1.456687

This Article is brought to you for free and open access by the Chemistry at DigitalCommons@URI. It has been accepted for inclusion in Chemistry Faculty Publications by an authorized administrator of DigitalCommons@URI. For more information, please contact digitalcommons-group@uri.edu. 


\title{
The quantum mechanics of cluster melting
}

\author{
Thomas L. Beck and J. D. Doll \\ Los Alamos National Laboratory, Los Alamos, New Mexico 87545 \\ David L. Freeman \\ Department of Chemistry, University of Rhode Island, Kingston, Rhode Island 02881
}

(Received 15 November 1988; accepted 27 January 1989)

\begin{abstract}
We present here prototype studies of the effects of quantum mechanics on the melting of clusters. Using equilibrium path integral methods, we examine the melting transition for small rare gas clusters. Argon and neon clusters are considered. We find the quantum-mechanical effects on the melting and coexistence properties of small neon clusters to be appreciable.
\end{abstract}

\section{INTRODUCTION}

The study of the equilibrium and dynamical properties of clusters is an active and growing area of research.' From a theoretical point of view, cluster studies are of interest for a variety of reasons. First, clusters themselves are important to a diverse range of physical phenomena and thus their study has a significant intrinsic interest. Moreover, the study of the size dependence of the physical properties of clusters is important in the analysis of the transition from finite to bulk behavior. Finally, clusters frequently play both a theoretical and practical role as prototype systems in the study of more general condensed phase and interfacial phenomena.

Theoretical studies of the equilibrium and dynamical cluster properties have typically utilized classical mechanical methods. Starting from a specified microscopic force law and the assumption of classical behavior, numerical Monte Carlo and molecular dynamics methods can produce estimates for the equilibrium and dynamical properties of such systems that are free of untestable numerical approximations. The studies of Lee, Barker, and Abraham ${ }^{2}$ of the thermodynamic properties of rare gas clusters are an excellent example of this type of investigation. For many problems, classical mechanical studies are appropriate and yield physically relevant predictions. For other problems, however, quantum-mechanical effects are sufficiently large that they cast doubt on the adequacy of purely classical approaches.

Numerical path integral methods are emerging as important tools for the analysis of many-body quantum-mechanical systems. ${ }^{3}$ These techniques have been shown to be useful in the study of a broad range of physical problems, including the study of the thermodynamic properties of clusters. The details of these approaches as well as their application to cluster problems have been outlined previously. ${ }^{4-6}$ It is sufficient to note here that these methods are the quantummechanical generalizations of conventional classical Monte Carlo methods. We also note that these methods have been extended recently to permit time-dependent, as well as equilibrium, applications. ${ }^{7-17}$

The present work is concerned principally with the cluster melting transition. The initial observation of a transition between solid-like and liquid-like forms for clusters was obtained via classical Monte Carlo and molecular dynamics simulations of argon clusters. ${ }^{18-23} \mathrm{~A}$ quantum statistical model was then developed by Berry and co-workers which is based on a harmonic oscillator-rigid rotor expression for the rigid form near melting and a model for the liquid form taken from nuclear theory. ${ }^{24-26}$ From these models, energy level spectra were constructed and a correlation diagram was drawn between the limiting spectra. The free energy was then determined as a function of temperature and a nonrigidity parameter describing motion along the correlation diagram. The implications of Refs. 25 and 26 are that finite clusters exhibit different and sharp melting and freezing temperatures, and that these temperatures approach each other and the bulk transition temperature as $N$, the number of atoms, approaches infinity. Between the melting and freezing temperatures, the model predicts a coexistence of liquid-like and solid-like forms in an ensemble of clusters, much as one would see in a chemical equilibrium between different isomeric forms. The model calculations were performed on hamiltonians constructed to characterize the melting behavior of $\mathrm{Ar}_{5}$. Classical simulations of the $\mathrm{Ar}_{13}$ cluster give evidence consistent with this approach in that a coexistence of solid-like and liquid-like forms, as determined by equilibrium and dynamical properties of the separate forms, is observed in the transition range of energies or temperatures in both isoergic and isothermal simulations. ${ }^{27.28}$

Further studies showed that all argon clusters with $N \geqslant 3$ exhibit a transition from a rigid, solid-like form to a nonrigid, liquid-like form as a function of energy, but the particular melting behavior of a given cluster is a very irregular function of $N . .^{29,30}$ For example, the argon clusters with $N=7,13$, and 19 exhibit especially high melting temperatures and show a range of energies over which one can distinguish a coexistence of solid-like and liquid-like forms. However, the $N=8,14$, and 20 clusters, resulting from the addition of a single argon atom to the "magic number" clusters, melt at much lower temperatures and exhibit no such coexistence. Also, $\mathrm{Ar}_{5}$ exhibits no coexistence ${ }^{31}$ and no cluster studied with $N>19\left(\mathrm{Ar}_{19}\right.$ has a relatively wide coexistence range) shows the coexistence in the transition region.

These findings led to a study of the physical mechanisms underlying the melting behavior. ${ }^{32,33}$ The study utilized periodic steepest descent quenches along relatively long trajectories in the transition range of energies to determine the potential minima accessed along a trajectory, the time scales for the motions, and the nature of the reaction paths linking the minima. The authors found that the conditions under which one can observe a coexistence of solid-like and liquidlike forms are the existence of a very stable potential minimum relative to the nearest accessible minima, and a separa- 
tion of time scales for the interwell and intrawell motions. The motions linking the minima are generally very collective motions; no clear "surface diffusion" of an outer atom on a rigid core was observed in the size range studied. The coexistence of solid-like and liquid-like forms refers to a coexistence of the motions about the very stable, low-lying minimum and the higher-lying, less stable minima, respectively, in cases where the potential surface has this form. Fully liquid-like behavior is observed when the time scale separation between intrawell and interwell motions breaks down. ${ }^{32-34}$

The conditions for coexistence are met in some but not all of the clusters between $N=3$ and 33, and are especially pronounced in the three "closed shell" pentagonal structures: the pentagonal bipyramid, the icosahedron, and the double icosahedron. However, systems like the $N=14,17$, and $N>19$ clusters have a large range of near-lying minima accessible in the transition region, and thus exhibit no coexistence of distinct liquid-like and solid-like forms. As observed in the quench studies, the number of near-lying minima accessible in the transition range for clusters with $N>19$ rises sharply with $N$, and thus the likelihood of observing coexistence of solid-like and liquid-like forms in the larger clusters due to the two well separated kinds of potential minima is small. One likely exception to this is the sequence of stable Mackay icosahedra at $N=55,147$, etc. ${ }^{35,36}$ Detailed knowledge of the potential surfaces of these small systems in terms of potential minima and connectivity is thus important in understanding the widely varying equilibrium and dynamical behavior of the clusters as a function of $N$.

Several experimental groups have begun to examine the possibility of cluster melting in pure and mixed clusters. Electron diffraction patterns have exhibited solid-like and liquid-like forms as a function of backing conditions in a molecular beam. ${ }^{37,38}$ Vibrational Raman spectroscopy on large clusters of $N_{2}$ (on the order of $10^{6}-10^{9}$ molecules) has been used to characterize gas-liquid, liquid-solid, and $\beta$ - to $\alpha$-solid phase transitions. ${ }^{39}$ Spectroscopic studies coupled with Monte Carlo and molecular dynamics simulations on mixed clusters of argon and a dopant species have revealed a very diverse behavior as a function of cluster size and temperature ${ }^{40-43}$ The spectra and simulations support the notion that one observes highly varying equilibrium and dynamical behavior as a function of the solvent cluster size and temperature. One of the studies ${ }^{42}$ discusses four distinct order-disorder transitions in carbazole-argon clusters.

The classical simulation studies leave unanswered questions concerning the magnitude of possible quantum-mechanical effects on the melting transition. Such effects could, in principle, be studied through the use of approximate methods; in practice, however, Monte Carlo path integral techniques are now sufficiently well developed that fully quantum calculations are quite feasible. In this paper we present numerically exact quantum calculations of the cluster melting transition of the $\mathrm{Ar}_{7}$ and $\mathrm{Ne}_{7}$ clusters. We find a small quantum effect for the argon cluster and a substantial quantum effect on the melting and coexistence properties for the neon cluster at temperatures along the melting curve. Studies examining quantum effects as a function of cluster size are currently underway.

Section II briefly sketches the numerical path integral methods appropriate for the present study and specifies the empirical potential model. Numerical results and associated discussions and applications are presented in Sec. III.

\section{FORMALISM}

This section describes the Monte Carlo path integral formalism used in the present study. General reviews outlining the application of both classical and quantum Monte Carlo methods to the study of clusters are available and should be consulted if a more detailed discussion is desired. ${ }^{4-6}$ For simplicity, a one-dimensional notation will be used in much of the following.

General thermodynamic averages for the canonical ensemble are given classically by

$$
\langle B\rangle=\int d x \exp [-\beta V(x)] B(x) / \int d x \exp [-\beta V(x)],
$$

where $B$ is some property of interest, $x$ is a (possibly) multidimensional variable denoting the coordinates necessary to describe the physical system, and $V(x)$ is the interaction potential. Averages of the type in Eq. (1) are not generally available analytically, except for certain idealized interaction potentials. They can, however, be evaluated by numerical Monte Carlo methods for general interactions. Classical Monte Carlo methods have been reviewed by Valleau and Whittington ${ }^{44}$ while issues germane to cluster applications have been discussed by Abraham. ${ }^{45}$

Thermodynamic averages for the canonical ensemble are given quantum mechanically by

$$
\langle B\rangle=\operatorname{Tr}[\exp (-\beta H) B] / \operatorname{Tr}[\exp (-\beta H)],
$$

where $H$ is the system Hamiltonian and the operator $B$ represents the property of interest. Considering the particular case where $B$ is diagonal in the coordinate representation, Eq. (2) takes on the form

$$
\langle B\rangle=\int d x\left\langle x\left|e^{-\beta H}\right| x\right\rangle B(x) / \int d x\left\langle x\left|e^{-\beta H}\right| x\right\rangle .
$$

The quantum-mechanical average is thus similar to the corresponding classical expression, except that the simple classical Boltzmann factor is replaced by the appropriate density matrix element.

Progress toward the general evaluation of quantum-mechanical averages of the type discussed above can be made using numerical path integral methods. The details of these approaches are described elsewhere. ${ }^{4-6}$ In simple terms, one transforms the formal "sum over paths" representation of the quantum-mechanical density matrix element in Eq. (3) into an ordinary, but high-dimensional integral over "auxiliary" variables that label the quantum-mechanical "paths". The details of this transformation depend on the particulars of how the paths are enumerated. The essential result, however, is that for the case of Boltzmann statistics, Eq. (3) can be written as

$$
\langle B\rangle=\int d x d \mathbf{a} \rho(x, \mathbf{a}) B(x) / \int d x d \mathbf{a} \rho(x, \mathbf{a}),
$$


where $\rho(x, a)$ is a known positive probability distribution function. The assumption of Boltzmann statistics is valid for the systems studied below. Within the Fourier path integral approach used in this study $\rho(x, a)$ is given by

$$
\rho(x, \mathbf{a})=\exp \left(-\sum_{k=1} \mathbf{a}_{k}^{2} / 2 \sigma_{k}^{2}-\beta(V)\right),
$$

where

$$
\begin{aligned}
& \sigma_{k}^{2}=2 \beta \hbar^{2} / m \pi^{2} k^{2}, \\
& \langle V\rangle=\int_{0}^{1} d u V[x(u)], \\
& x(u)=x+\sum_{k=1} \mathbf{a}_{k} \sin (k \pi u) .
\end{aligned}
$$

The quantum-mechanical expression, Eq. (4), is now an average over both the original coordinates $x$ and the associated auxiliary variables, $a$. This average can, however, be evaluated using standard Monte Carlo methods. In essence, the numerical path integral approach has transformed the original quantum-mechanical problem in $x$ into a "classical" problem in the augmented space $(x, a)$. In practice, one typically truncates the formally infinite Fourier representation of the paths, Eq. (6c), at some upper limit $k_{\max }$ following convergence of the quantity of interest. The value of $k_{\max }$ required for any particular problem can be estimated by requiring that the natural length scale for that Fourier variable, Eq. (6a), be small relative to the length scale of the interaction potential. Useful techniques designed to improve the convergence of the basic Fourier approach are available and were used in the present work. ${ }^{46,47} \mathrm{~A}$ number of cluster studies have been reported using the methods described above as well as related approaches. ${ }^{4-6,48-50}$

In the present work we are particularly concerned with possible quantum-mechanical effects on the transition between solid-like and liquid-like behavior in clusters. We have investigated this issue by computing the root-meansquare ( $\mathrm{rms}$ ) bond length fluctuations in the cluster, defined as

$$
\delta=\frac{2}{N(N-1)} \sum_{i<j} \frac{\sqrt{\left(\left\langle r_{i j}^{2}\right\rangle-\left\langle r_{i j}\right)^{2}\right.}}{\left\langle r_{i j}\right\rangle},
$$

as a function of temperature both classically and quantum mechanically. The indices in the sum in Eq. (7) range over the particles within the cluster. The rms bond length fluctuations were evaluated by both classical and path integral Monte Carlo methods. This quantity gives a clear indication of the onset of nonrigid or liquid-like motions in the cluster, as it rises sharply at energies or temperatures where the cluster begins to exhibit, for example, diffusive modes in the power spectrum of the velocity autocorrelation function and a linearly increasing mean-square displacement. ${ }^{27-30,32,33}$ The rapid rise of $\delta$ at the melting point of the clusters is reminiscent of the Lindemann criterion for bulk melting, which states that the bulk material melts when the fluctuations reach $10 \%-15 \%$.

The second quantity we examine is the mean potential energy of the cluster. This quantity is calculated classically as in Eq. (1) and quantum mechanically via Eq. (4). We calculate the average potential energy over short intervals along the Metropolis walk and then calculate the distribution of the short interval averages. The distribution gives an indication of the "basins of attraction" in the configuration space in the transition region. This calculation is analogous to the calculation of the distribution of coarse grained kinetic energies in molecular dynamics studies. ${ }^{27-30}$ However, we emphasize that the "time" along the Monte Carlo procedure is fictitious, and that the distribution merely gives some indication of the nature of the potential surface accessed along the Metropolis walk. A similar averaging procedure was used in Ref. 28 in a study of the constant temperature behavior of the $\mathrm{Ar}_{13}$ cluster; coexistence of solid-like and liquidlike forms is indicated by the observation of a bimodal form for the distribution. In effect, the distribution of potential energies gives a mapping of the potential minima accessed along the Metropolis walk, as long as the walk is trapped for appreciable amounts of time along the Monte Carlo integration in motions about each of the minima. The seven atom cluster has a potential surface which leads to coexistence of forms in the transition region in classical simulations; that is, it possesses one low-lying minimum (the pentagonal bipyramid) relative to the higher-lying minima. ${ }^{32-35}$

In the present study the cluster potential was approximated by a pairwise sum of Lennard-Jones interactions. Table I lists the well depth and range parameters used for the different rare gases in the present study. In addition to these pairwise interactions, the cluster potential used in the present study included a spherical "confining" potential $V_{c}$ of the form

$$
V_{c}=\sum_{i}\left(r_{i} / R_{c}\right)^{20}
$$

where the parameter $R_{c}$ specifies the cluster confining volume. The radius of the confining potential was chosen to be much larger than the radius of the cluster in these studies to mimic free boundary conditions; typically, a value of $20 \mathrm{a} . \mathrm{u}$. was used. Varying the radius to larger values had no noticeable effect on the $\delta$ or mean potential values.

Since the potential of interaction between the atoms is of a pairwise, Lennard-Jones form, the law of corresponding states for the various rare gases holds in classical simulations of a given cluster size if free boundary conditions are assumed. In practice, the confining potential was set to a large enough value that the system experienced essentially free boundary conditions; test calculations were performed on the neon and argon clusters which showed that the law of corresponding states holds. Thus, the rms bond length fiuctuation curves are the same for classical $\mathrm{Ar}_{7}$ and $\mathrm{Ne}_{7}$ when the $\delta$ values are plotted as a function of the temperature scaled by the well depth. The quantum results of course do not necessarily obey this rule.

TABLE I. Lennard-Jones parameters used in the neon and argon studies.

\begin{tabular}{lrl}
\hline \hline & $\epsilon$ & $\sigma$ \\
\hline Neon & $36.2 \mathrm{~K}$ & $2.74 \AA$ \\
Argon & $119.4 \mathrm{~K}$ & $3.41 \AA$ \\
\hline \hline
\end{tabular}




\section{RESULTS AND DISCUSSION}

The methods of Sec. II were applied to the study of the melting transition in seven atom argon and neon clusters. The results for the quantum and classical calculation of the rms bond length fluctuations as a function of scaled temperature are summarized in Fig. 1. Except in the low-temperature region, the classical and quantum-mechanical results for argon are quite similar. The differences between the quantum and classical results at the low temperatures arise from the zero-point motions about the pentagonal bipyramidal structure. There is little or no quantum effect on the melting temperature, however.

This is not the case for neon, where appreciable differences between the classical and quantum results are visible in the transition region of Fig. 1. The quantum-mechanical melting curve for neon is shifted to lower temperatures relative to the classical calculation. This result can be explained as follows. The introduction of quantum mechanics has two effects: zero-point motion and tunneling. If, for descriptive purposes, we view the potential surface for the quantum case as a modified potential of the Feynman-Hibbs form ${ }^{51}$ (which corresponds to a local gaussian average over length scales proportional to the thermal deBroglie wavelength), the local averaging has the effect of raising the minima and lowering the saddles of the multidimensional surface. Both of these effects contribute to the onset of nonrigid behavior at lower temperatures than is observed in the classical calculation.

The bulk melting temperature of neon $(23.48 \mathrm{~K})$ is very close to that predicted by assuming the law of corresponding states and scaling the melting temperature of bulk argon $(83.95 \mathrm{~K})$, which can to a high degree of accuracy be treated

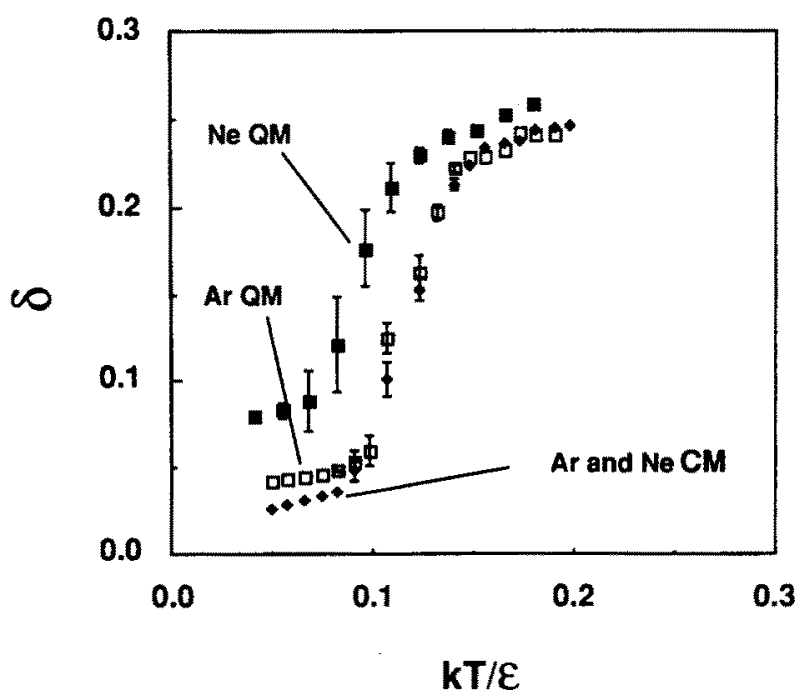

FIG. 1. Shown are the classical and path integral Monte Carlo estimates of the rms bond length fuctuations [Eq. (7)] for seven atom argon and neon clusters. The results for each study utilized $4 \times 10^{6}$ Monte Carlo points for temperatures in the transition region and smaller numbers of points elsewhere. The quantum-mechanical studies used Fourier path integral methods with gradient partial averaging. One (five) Fourier coefficients were used in the quantum-mechanical argon (neon) studies. Convergence in the low-temperature results was obtained with this number of coefficients. classically, so quantum effects on melting are not large in the bulk neon system. ${ }^{52}$ Therefore, the shift of the $\delta$ curve in the cluster is a finite size effect due to the accessibility of neighboring potential minima at lower temperatures in the cluster relative to the bulk.

Also, note that the quantum rms fluctuations are substantial for the neon clusters even as the temperature approaches $0.0 \mathrm{~K}$. The zero-point motions are large enough $(\delta \approx 0.075)$ that the system accesses anharmonic regions of the potential surface even at very low temperatures. In fact, the quantum calculation gives a $\delta$ value at low temperatures which is very close to the value where transitions between various potential minima begin to occur in the classical simulation.

The distributions of potential energies accessed along the Metropolis walk have also been calculated (Fig. 2) for the neon and argon clusters, as discussed in Sec. II. The classical results are very similar to the behavior observed in Monte Carlo simulations of $\mathrm{Ar}_{13}{ }^{28}$ At low temperatures, a unimodal distribution is observed as the Metropolis walk samples states corresponding to motions about the stable pentagonal bipyramidal structure. As the temperature is increased into the transition range, the distribution takes on a bimodal form which is consistent with earlier molecular dynamics simulations of the seven atom system ${ }^{29}$ and Monte Carlo simulations of the 13 atom cluster. ${ }^{28}$ The low potential-energy structure corresponds to the pentagonal bipyramid, while the peak at higher potential-energy results from motions about the higher-lying minima. The coexistence of motions within the stable pentagonal bipyramidal well and motions among the higher-lying minima was interpreted as solid-liquid coexistence in the dynamics simulations of $\mathrm{Ar}_{7},{ }^{29,34}$ since the dynamical properties of the two forms indicate a separation into solid-like and liquid-like properties, respectively.

The quantum effects on the distributions for the argon cluster are not large. The same bimodal distribution is observed in the transition range of temperatures, but the quantum results are shifted to slightly higher potential energy. For neon, however, the alteration of the distribution is dramatic. At all temperatures studied, the quantum distribution takes on a broad unimodal form; the coexistence behavior [as indicated by a clear bimodal form for the distribution, as in Figs. 2(e) and 2(g) ] resulting from the particular form of the potential surface in the classical simulation is lost. Also, the quantum distribution is shifted to much higher potential energies. This occurs even at $2.0 \mathrm{~K}$ where the classical distribution is a very narrow unimodal distribution resulting from sampling about the most stable, solid-like structure. In effect, the neon atoms at these temperatures have a large enough thermal deBroglie wavelength that the cluster is very "soft," and the potential surface is explored in a very different manner than in the classical system. Even at very low temperatures, the cluster accesses regions of the potential surface which, for the classical system, would allow passage over saddles to neighboring potential minima. As mentioned above, if one views the potential surface for the quantum neon cluster as a modified potential of the Feynman-Hibbs form, the averaging over length scales proportional to the 

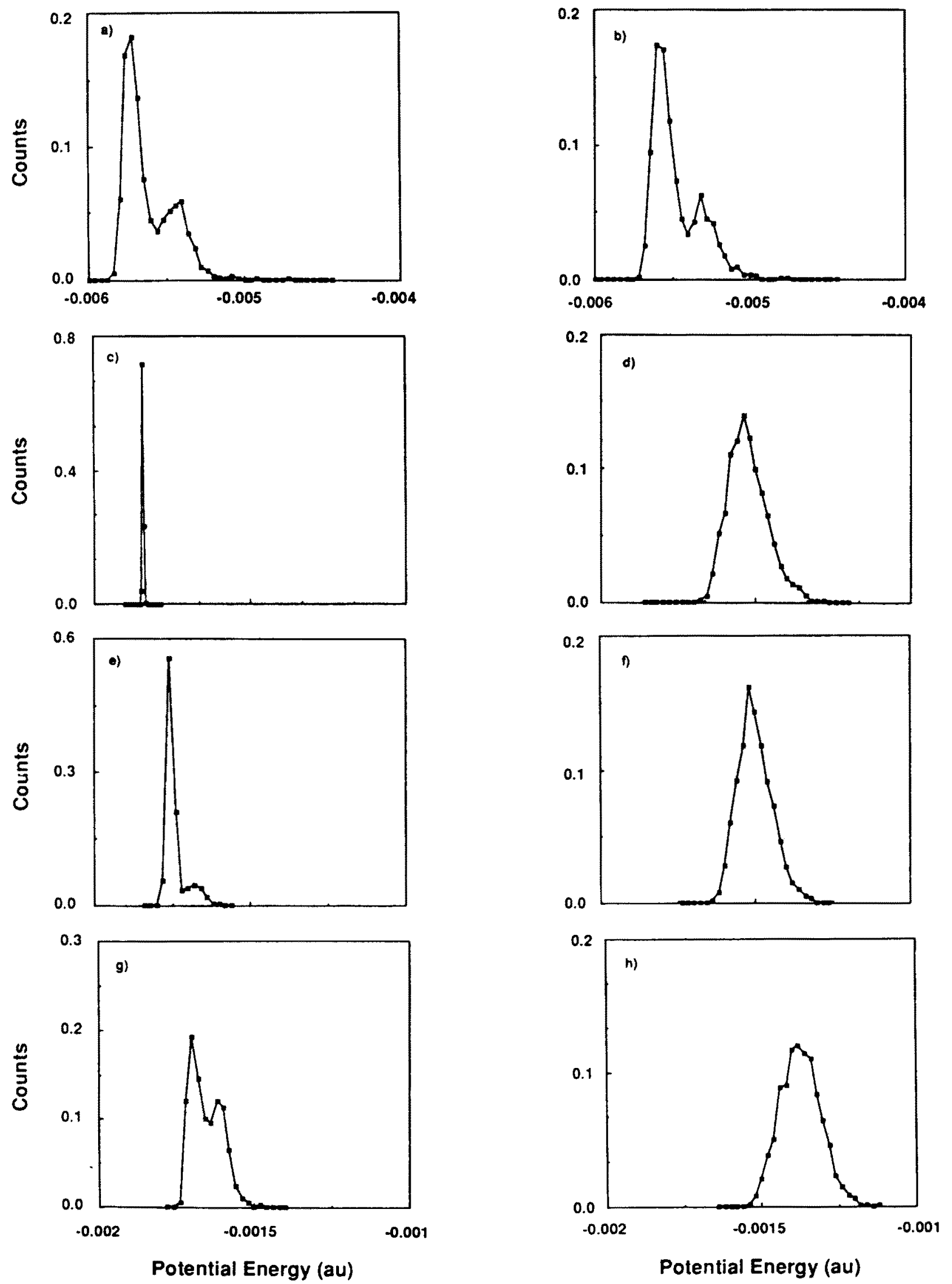

FIG. 2. Distributions of short time averaged potential energies for the classical and quantum simulations calculated along long particle procedures (typically $2 \times 10^{6}$ attempted moves). The averaging interval was taken to be 7000 attempted particle moves, with approximately a $50 \%$ acceptance rate at each temperature. For the quantum results, particle and Fourier coefficient moves were attempted at each step. (a) Classical Ar, at $19.0 \mathrm{~K}$, in the transition range of temperatures. (b) Quantum $\mathrm{Ar}_{7}$ at $19.0 \mathrm{~K}$. (c) Classical $\mathrm{Ne}_{7}$ at $2.0 \mathrm{~K}$. (d) Quantum $\mathrm{Ne}_{7}$ at $2.0 \mathrm{~K}$. (e) $\mathrm{Classical} \mathrm{Ne}_{7}$ at $5.0 \mathrm{~K}$. (f) Quantum $\mathrm{Ne}_{7}$ at $3.0 \mathrm{~K}$. (g) Classical $\mathrm{Ne}_{7}$ at $6.5 \mathrm{~K}$. (h) Quantum $\mathrm{Ne}_{7}$ at $6.5 \mathrm{~K}$. 
deBroglie wavelength significantly alters the nature of the potential surface.

Several experiments have now been done on cluster systems composed of a dopant species either imbedded in or adsorbed on argon clusters. ${ }^{40-43} \mathrm{~A}$ wide range of spectral behavior has been observed for given cluster sizes and temperatures. The results discussed above imply that, for experiments probing the transition from solid-like to liquid-like behavior in the analogous neon clusters, quantum effects can be expected to be important. We are currently investigating such mixed systems.

Quantum Monte Carlo methods provide a useful tool for the calculation of properties which are indicators of the onset of nonrigid behavior in small clusters. The quantum effects on the melting and solid-like, liquid-like coexistence properties of the clusters are found to be relatively small for argon and large for neon. Other cluster phenomena that would be amenable to study by these methods are, e.g., mass segregation in clusters due to quantum effects, the structure of species adsorbed on the surface of a cluster, and the stability of various clusters formed in a nucleation process. Newly developed time-dependent methods ${ }^{7-17}$ should prove useful in calculating dynamical properties of clusters and other many-body systems. Work is currently underway utilizing these methods.

Note added in proof. Following completion of this paper, we became aware of a recent path integral simulation of small argon clusters by Franke et al..$^{53}$

\section{ACKNOWLEDGMENTS}

One of us (D.L.F.) acknowledges the donors of the Petroleum Research Fund, administered by the American Chemical Society, for partial support of this research. This work was also supported in part by grants from Research Corporation.

'See, for example, The Physics and Chemistry of Small Clusters, edited by $\mathrm{P}$. Jena, B. K. Rao, and S. N. Khanna (Plenum, New York, 1987).

${ }^{2}$ J. K. Lee, J. A. Barker, and F. F. Abraham, J. Chem. Phys. 58, 3166 (1973).

${ }^{3}$ For a cross section of recent activity in this area, see the Proceedings of the Metropolis Quantum Monte Carlo Conference, published in J. Stat. Phys. 43, 729-1244 (1986).

${ }^{4}$ D. L. Freeman and J. D. Doll, J. Chem. Phys. 80, 5709 (1984).

${ }^{5}$ D. L. Freeman and J. D. Doll, J. Chem. Phys. 82, 462 (1985).

${ }^{6} \mathrm{D}$. L. Freeman and J. D. Doll, in Advances in Chemical Physics, edited by I. Prigogine and S. A. Rice (Wiley, New York, 1988), Vol. 70B, p. 139.

${ }^{7} J$. D. Doll, J. Chem. Phys. 81, 3536 (1984)

${ }^{8}$ V. S. Filinov, Nucl. Phys. B 271, 717 (1986).

'J. D. Doll and D. L. Freeman, in Advances in Chemical Physics, edited by I. Prigogine and S. A. Rice (Wiley, New York, 1989), Vol. 73, p. 289.

${ }^{10}$ J. D. Doll, R. D. Coalson, and D. L. Freeman, J. Chem. Phys. 87, 1641 (1987).
"J. Chang and W. H. Miller, J. Chem. Phys. 87, 1648 (1987).

${ }^{12}$ N. Makri and W. H. Miller, Chem. Phys. Lett. 139, 10 (1987).

${ }^{13}$ J. D. Doll, D. L. Freeman, and M. J. Gillan, Chem. Phys. Lett. 143, 277 (1988).

${ }^{14}$ N. Makri and W. H. Miller, J. Chem. Phys. 89, 2170 (1988).

${ }^{15}$ J. D. Doll, T. L. Beck, and D. L. Freeman, J. Chem. Phys. 89, 5753 (1988).

${ }^{16}$ T. L. Beck, J. D. Doll, and D. L. Freeman, J. Chem. Phys. 90, 3181 (1989).

${ }^{17}$ R. E. Cline and P. G. Wolynes, J. Chem. Phys. 88, 4334 (1988).

${ }^{18}$ C. L. Briant and J. J. Burton, J. Chem. Phys. 63, 2045 (1975).

${ }^{19}$ R. D. Etters and J. B. Kaelberer, Phys. Rev. A 11, 1068 (1975).

${ }^{20}$ J. B. Kaelberer and R. D. Etters, J. Chem. Phys. 66, 3223 (1977).

${ }^{21}$ R. D. Etters and J. B. Kaelberer, J. Chem. Phys. 66, 5112 (1977).

${ }^{22}$ D. J. McGinty, J. Chem. Phys. 58, 4733 (1973).

${ }^{23}$ W. D. Kristensen, E. J. Jensen, and R. M. J. Cotterill, J. Chem. Phys. 60, 4161 (1974).

${ }^{24}$ G. Natanson, F. Amar, and R. S. Berry, J. Chem. Phys. 78, 399 (1983).

${ }^{25}$ R. S. Berry, J. Jellinek, and G. Natanson, Chem. Phys. Lett. 107, 227 (1984).

${ }^{26}$ R. S. Berry, J. Jellinek, and G. Natanson, Phys. Rev. A 30, 919 (1984)

${ }^{27}$ J. Jellinek, T. L. Beck, and R. S. Berry, J. Chem. Phys. 84, 2783 (1986).

${ }^{28}$ H. Davis, J. Jellinek, and R. S. Berry, J. Chem. Phys. 86, 6456 (1987).

${ }^{29}$ T. L. Beck, J. Jellinek, and R. S. Berry, J. Chem. Phys. 87, 545 (1987).

${ }^{30}$ T. L. Beck, D. M. Leitner, and R. S. Berry, J. Chem. Phys. 89, 1681 (1988).

${ }^{3}$ T. L. Beck ( unpublished)

${ }^{32}$ T. L. Beck and R. S. Berry, in The Physics and Chemistry of Small Clusters, edited by P. Jena, B. K. Rao, and S. N. Khanna (Plenum, New York, 1987).

${ }^{33}$ T. L. Beck and R. S. Berry, J. Chem. Phys, 88, 3910 (1988).

${ }^{34}$ F. Amar and R. S. Berry, J. Chem. Phys. 85, 5943 (1986).

${ }^{35}$ M. R. Hoare, in Advances in Chemical Physics, edited by I. Prigogine and S. A. Rice (Wiley, New York, 1979), Vol. 40.

${ }^{36}$ J. D. Honeycutt and H. C. Andersen, J. Phys. Chem. 91, 4950 (1987).

${ }^{37}$ E. J. Valente and L. S. Bartell, J. Chem. Phys. 80, 1458 (1984).

${ }^{38}$ J. M. Farges, M. F. deFaroudy, B. Raoult, and G. Torchet, J. Chem. Phys. 78, 5067 (1983)

${ }^{39}$ R. Beck and J. W. Nibler, Chem. Phys. Lett. 148, 271 (1988).

${ }^{40}$ T. E. Gough, D. G. Knight, and G. Scoles, Chem. Phys. Lett. 97, 155 (1983).

${ }^{41}$ D. Eichenauer and R. J. LeRoy, J. Chem. Phys. 88, 2898 (1988).

${ }^{42}$ S. Leutwyler and J. Bosiger, in Large Finite Systems, edited by J. Jortner, A. Pullman, and B. Pullman (Reidel, Boston, 1987).

${ }^{43}$ M. Y. Hahn and R. L. Whetten, Phys. Rev, Lett. 61, 1190 (1988).

${ }^{44} \mathrm{~J}$. P. Valleau and S. G. Whittington, in Modern Theoretical Chemistry, edited by B. J. Berne (Plenum, New York, 1977), Vol. 5, pp. 137-168.

${ }^{45}$ F. F. Abraham, Homogeneous Nucleation Theory (Academic, New York, 1974).

${ }^{46}$ J. D. Doll, R. D. Coalson, and D. L. Freeman, Phys. Rev. Lett. 55, 1 (1985).

${ }^{47}$ R. D. Coalson, D. L. Freeman, and J. D. Doll, J. Chem. Phys. 85, 4567 (1986).

${ }^{48}$ M. Marchi, M. Sprik, and M. L. Klein, J. Chem. Phys. 89, 4918 (1988).

${ }^{49} \mathrm{G}$. J. Martyna and B. J. Berne, J. Chem. Phys. 88, 4516 (1988).

${ }^{50}$ R. N. Barnett, U. Landman, C. L. Cleveland, and J. Jortner, Chem. Phys. Lett. 145, 382 (1988).

${ }^{5}$ R. P. Feynman and A. R. Hibbs, Quantum Mechanics and Path Integrals (McGraw-Hill, New York, 1965).

${ }^{52}$ See, for example, a quantum path integral calculation on liquid neon: $D$. Thirumalai, R. W. Hall, and B. J. Berne, J. Chem. Phys. 81, 2523 (1984).

${ }^{53}$ G. Franke, E. Hilf, and L. Polley, Z. Phys. D 9, 343 (1988). 
The Journal of Chemical Physics is copyrighted by the American Institute of Physics (AIP). Redistribution of journal material is subject to the AIP online journal license and/or AIP copyright. For more information, see http:/ojps.aip.org/jcpo/jcpcr/jsp Copyright of Journal of Chemical Physics is the property of American Institute of Physics and its content may not be copied or emailed to multiple sites or posted to a listserv without the copyright holder's express written permission. However, users may print, download, or email articles for individual use. 
The Journal of Chemical Physics is copyrighted by the American Institute of Physics (AIP). Redistribution of journal material is subject to the AIP online journal license and/or AIP copyright. For more information, see http://ojps.aip.org/jcpo/jcper/jsp 